\title{
Play dynamics on electronic gaming machines: A conceptual review
}

\author{
PAUL DELFABBRO* and DANIEL L. KING \\ School of Psychology, University of Adelaide, Adelaide, SA, Australia
}

(Received: October 18, 2018; revised manuscript received: January 31, 2019; accepted: March 15, 2019)

\begin{abstract}
Background and aims: This paper proposes that future research into electronic gaming machines (EGMs) is likely to benefit from conceptual and methodological approaches that capture the dynamic interplay between game parameters as well between the psychological needs of gamblers and their behavior. Methods: The argument concerning the importance of player dynamics is developed in two sections. The first involves an analysis of existing work, which investigates individual gaming machine features and then a discussion; the second reappraises the value of Apter's (1982) Reversal Theory as a framework for understanding behavioral dynamics and the interplay between gambler's need states and their play choices. Results: It is argued that existing methods based on the modification of single features are going to be limited and that differences in observed behavior may relate to measurable differences in motivational states before and during gambling sessions. Discussion and conclusions: It is concluded that a more dynamic and interactive approach to studying EGMs could be facilitated by innovations in Big Data and greater access to genuine player data. It is argued that such work may help to inform in situ research methods as well as clinical interventions for gamblers at risk or those already involved in interventions involving exposure and controlled gambling.
\end{abstract}

Keywords: electronic gaming machines, play dynamics, gambling behavior, motivation

\section{INTRODUCTION: SLOT-MACHINE GAMBLING}

"Spinning reel" slot machines are one of the most lucrative forms of gambling in the world. In these games, players place bets on the outcomes of spinning reels (often 3-5) to obtain combinations of winning symbols. Such games can be based on mechanical reels, be offered online, or through complex electronic gaming machines (EGMs) or videolottery terminals. With most modern machines, players are given opportunities to bet on different play lines and also vary the amount they bet per line. Players can also play quickly, often with only 3-4 s between each separate game. As a result, EGMs offer players one of the most rapid and continuous forms of gambling. With relatively simple operational rules, low entry place, and highly appealing modern audio-visual displays, these machines have been shown to have appeal across a wide range of demographic groups and are the highest earning form of gambling in many countries (Dow Schüll, 2012; Productivity Commission, 2010; Queensland Treasury, 2018).

International gaming statistics show that there at least seven million active gaming machines in the world, with the highest total count in Japan. The US has 869,000; the UK 167,000; Canada 100,000; and there are almost 200,000 EGMs in Australia (Queensland Treasury, 2018). Countries considerably vary in the number of EGMs per capita. Italy has the lowest population per machine ratio (136 persons per machine) and Australia is third with 121 persons per machine (Ziolkowski, 2016). Machines are generally more sparsely observed in the UK (384 people per machine) and in Canada (359 people per machine). EGMs are a particular area of policy concern in countries such as Australia and New Zealand (Queensland Treasury, 2018) because of the high prevalence per capita of high-intensity machines that allow $3.5 \mathrm{~s}$ spin speeds and stake sizes of up to \$AUS10. In these countries (Productivity Commission, 2010), EGMs are the activity most likely to be associated with problem gambling. Figures drawn from prevalence studies in Australia as well as treatment-seeking populations consistently show that EGMs account for around $70 \%$ cases of adult problem gambling and remain the predominant $(90 \%)$ type of gambling reported by women who report gambling problems (Armstrong \& Carroll, 2017; Armstrong, Thomas, \& Abbott, 2017; Delfabbro, 2017; Productivity Commission, 2010). Such observations have led to considerable research interest in understanding why EGMs are so likely to be harmful to a significant proportion of regular players (estimates suggest around 1 in 5; Livingstone, 2005; Productivity Commission, 2010). It has also fueled strong community and advocacy against EGMs, particularly because EGM gambling attracts a disproportionate amount of revenue from lower socioeconomic groups and problem gamblers (Dowling, Smith, \& Thomas, 2005; Livingstone, 2001, 2005;

\footnotetext{
* Corresponding author: Prof. Paul Delfabbro; School of Psychology, University of Adelaide, North Terrace, Adelaide, SA 5005, Australia; Phone: +61 88313 4936; E-mail: Paul.delfabbro@ adelaide.edu.au
}

This is an open-access article distributed under the terms of the Creative Commons Attribution-NonCommercial 4.0 International License, which permits unrestricted use, distribution, and reproduction in any medium for non-commercial purposes, provided the original author and source are credited, a link to the CC License is provided, and changes - if any - are indicated. 
Livingstone, Woolley, Zazryn, Bakacs, \& Shami, 2008; Productivity Commission, 1999).

Such concerns have led to the development of dedicated research that attempted to identify the structural characteristics of EGMs that influence player behavior and most strongly contribute to excessive gambling and gamblingrelated harm. Much of this work has shown that EGM gambling involves a complex interplay between players and the machines. Some people seem to possess vulnerabilities that make them more likely to experience harm associated with EGMs and machines clearly possess several characteristics that make them more likely to encourage harmful levels of play (Parke, Parke, \& Blaszczynski, 2005). Evidence supporting this proportion has derived most commonly from well-controlled experimental studies that manipulate single machine characteristics (e.g., frequency of near misses, win frequencies, or win sizes) to examine how this influences the behavior (e.g., bet sizes and behavioral persistence). Although we believe that this work is valuable and has strong internal validity, researchers are now increasingly facing situations involving large real-time data sets derived from industry where one may be faced with a complex interplay of multiple variables. As a result, we argue that it is early to review how existing methods and conceptual approaches (usually based on single structural characteristics and single playing sessions) may need to be complemented by new conceptual approaches. In real-life gambling data sets, multiple and often highly correlated variables may be operating simultaneously. In addition, how players react to the machines may vary over time and across sessions based on changes in the individual themselves (e.g., their mood) and outcomes in previous sessions. Accordingly, this paper attempts to advance conceptual approaches that might address each of these two concerns in turn.

In this first part of the paper, which focuses on the dynamics of machines, we argue that existing static or experimental approaches to studying EGMs probably cannot fully capture the full complexity and two-way dynamics of actual EGM gambling in situ. We summarize the important advances in existing experimental research as well as the small number of studies, which have attempted to examine the effects of modifying EGMs on real-life gambling behavior. It will be argued that valid policy-relevant findings need to be informed by studies, which can capture the complex interdependencies between different aspects of game play (e.g., bet size, play duration, and reinforcement type). As we show, without such analysis, there can be a danger that modifications to individual features might be advanced as policy responses to reducing gambling-related harm, without reference to how these changes might be undermined by changes to other related parameters. In this paper, we focus specifically on issues relating to the cost of gambling because these are: (a) most strongly related and therefore potentially confounded and (b) because Parke et al. (2015) identify features associated with the "cost of gambling" as having some of the strongest implications for reducing harm.

In the second part of the paper, which focuses on the dynamics of players, we examine the issue of within play dynamics and the potential need to consider conceptual approaches that capture changes in player-machine interactions over time. In this section, we note that there are many research findings that document that motivations and "need states" (e.g., mood and need for arousal) that underline EGM gambling. We argue that, while many of these findings are useful, they could be extended to capture a more dynamic understanding of how player motivational states might influence behavior. Drawing upon recent research by Dixon et al. (2014) and Parke et al. (2015), we will argue that a player's choice of machine as well as how they play within session is likely to reflect a dynamic interplay between motivational and mood states, behavior, and outcomes. Players are likely to choose machines and have playing "signatures" that satisfy their motivations and needs and these may change over time and within session. In light of this, we argue that there may be value in reconsidering Anderson and Brown's (1987) earlier suggestion that gambling could be usefully studied using frameworks that recognize the dynamic and varying nature of motivational states, including Apter's (1982) Reversal Theory (RT).

\section{MACHINE FEATURES AND THEIR ROLE IN GAMBLING-RELATED HARM}

Attempts to reduce harm associated with EGMs have generally taken one of two approaches: the supply- or demand-side approaches. The first, or supply side, approach focuses on the nature of the product. It is predicated on the assumption that EGMs are inherently risky products that need to be modified to make them safer (Doran \& Young, 2010; Dow Schüll, 2012; Livingstone et al., 2008; Parke et al., 2015). Accordingly, the aim is to identify machine features, which may contribute to harmful levels of gambling and then modify or eliminate these features. Researchers then look for measurable changes in outcomes that might signify modifications in behavior or reductions in harm; such indicators may include a reduction in problem gambling prevalence, reduced expenditure or time spent, or fewer reports of harm associated with EGMs. Many such machine features have been described in detail by Dow Schüll in the book Addiction by Design and also more recently by Parke et al. (2015). Although there is little question that industry designers have put considerable work into designing machines, which (in their minds) will yield maximum time on machine and revenue, there arises another question as to whether such features: (a) have a meaningful effect on behavior and (b) allow one to identify machines that pose the greatest risk to players.

These observations are outlined by Parke et al.'s (2015) report that reviews categories of EGM features and the research evidence in support of their impact on behavior and problem gambling. As indicated in Table 1, most of these relate to factors that influence actual game play, whereas others relate more to how one inputs money into the machine or what information is made available to players. Parke et al. conclude that all these features are worthy of consideration and may play a role in maintaining player interest or expenditure, but that evidence in support of the harm-minimization benefit of modifying or removing many features is mixed. In other words, although there is a 
Table 1. Gaming machine features identified in harm-minimization research

\begin{tabular}{|c|c|c|}
\hline Category & Examples & Comments \\
\hline Game characteristics & $\begin{array}{l}\text { Free spins/bonus features } \\
\text { Hold features }\end{array}$ & $\begin{array}{l}\text { Players like bonus features, but not clear if implicated as a } \\
\text { factor in problem gambling }\end{array}$ \\
\hline Ambient characteristics & $\begin{array}{l}\text { Sound, colors, and lights } \\
\text { Screen/seat design }\end{array}$ & $\begin{array}{l}\text { Likely to make machines more popular, but unclear if } \\
\text { related to problem gambling }\end{array}$ \\
\hline Speed and frequency of gambling & $\begin{array}{l}\text { Play speed } \\
\text { Frequent rewards } \\
\text { Continuity }\end{array}$ & $\begin{array}{l}\text { Likely to make modern machines popular in general and } \\
\text { potentially problematic, but most machines have these } \\
\text { qualities }\end{array}$ \\
\hline Reward characteristics & $\begin{array}{l}\text { Volatility } \\
\text { Big wins vs. smaller wins } \\
\text { Near misses } \\
\text { Losses disguised as wins } \\
\text { Jackpots }\end{array}$ & $\begin{array}{l}\text { Features are common to many machines and some } \\
\text { evidence that problem gamblers are more responsive to } \\
\text { some of these features }\end{array}$ \\
\hline Cost of gambling & $\begin{array}{l}\text { Return to player } \\
\text { Available bet size and playing lines }\end{array}$ & $\begin{array}{l}\text { Ability to spend money quickly strongly implicated as a } \\
\text { factor in excessive expenditure by problem gamblers }\end{array}$ \\
\hline Payment and accounting & $\begin{array}{l}\text { Playing with credit } \\
\text { Note acceptors } \\
\text { Access to credit/cash withdrawals }\end{array}$ & $\begin{array}{l}\text { Easy access to money strongly implicated as a factor } \\
\text { implicated in excessive expenditure by problem gamblers }\end{array}$ \\
\hline Messaging/product information & $\begin{array}{l}\text { Information on odds/return to player/ } \\
\text { randomness } \\
\text { Responsible gambling messaging }\end{array}$ & $\begin{array}{l}\text { Some evidence that more self-reflective and dynamic } \\
\text { messages may be useful, but not a lot of strong evidence } \\
\text { in support of efficacy for problem gamblers }\end{array}$ \\
\hline
\end{tabular}

Note. Adapted from Parke et al. (2015).

body of research that has considered the role of play speeds, sounds, graphics, the availability of free spin, or returns to player on player behavior, none of this work provides persuasive evidence that modifications to these features would be likely to reduce gambling harm. Instead, the strongest evidence for harm minimization relates to the monetary aspects of gambling: how easily people can find money to gamble and have it credited on the machine and how rapidly they can spend money. For example, evidence would support the view that allowing people to feed in banknotes, access bank accounts, or bank machines near or in the proximity of the machine is more likely to contribute to greater expenditure (Parke et al., 2015). Similarly, machines that allow people to bet larger amounts on many lines (multiline machines) more likely encourage longer playing sessions and/or higher bets per game (Parke et al., 2015).

The second, or demand side, approach focuses on the gambler. Such approaches strike to encourage safer, more "responsible," or less harmful gambling behavior, which is typically defined as gambling that involves a level of monetary and time commitment that is less likely to lead to harm (Neal, Delfabbro, \& O’Neil, 2005). Two categories of machine characteristic in Table 1 clearly fall into this category. Anything that diminishes people's access to money in EGMs or how much can be spent per play or over a period of time is largely based on the behavior of gamblers. Similarly, when one provides messages or information gamblers, this does not change the machine, but tries to modify what the gambler does. Other categories would relate to supply side (or machine changes), which are designed to influence behavior: either directly or indirectly. For example, restricting how fast people can play, the number of betting lines, the bet per line, or maximum bet may serve to constrain people behavior within certain boundaries. Others such as changing the aesthetics (sounds or light) or bonus features would have more indirect effects if it influenced people's interest in the game.

In our view, this area is fraught with many conceptual challenges. The first issue is to do with the popularity of features. Some features (e.g., free spins) appealing graphics and sound clearly encourage people to play EGMs. However, these features may appeal to all players and not just problem gamblers. Therefore, they may not be features, which necessarily increase the likelihood of some people becoming problem gamblers. The second issue relates to the degree of modification. Some suggested modifications refer to the complete removal of a feature (e.g., autoplay and banknote acceptors), whereas others only constrain the behavior (e.g., bet limits). A third issue is the universality of the modification. Some policies, for example, removal of autoplay would affect all machines, whereas reducing the availability of certain jackpot features might only be relevant to the class of machines that contain that feature. Other machines may remain unaffected by the policy.

The ultimate aim of game feature analyses is to identify what can reduce the harm from EGMs, but this is likely to yield increasingly diminished returns as machines become increasingly homogenous and more technologically sophisticated. It may still be possible to say that a particular class of gambling product is potentially more harmful than others, or differentiate between quite different classes or generations of machines, but this process may be harder to perform within categories of product. This is illustrated in Figure 1, which highlights the diminishing variability of gaming machines. 


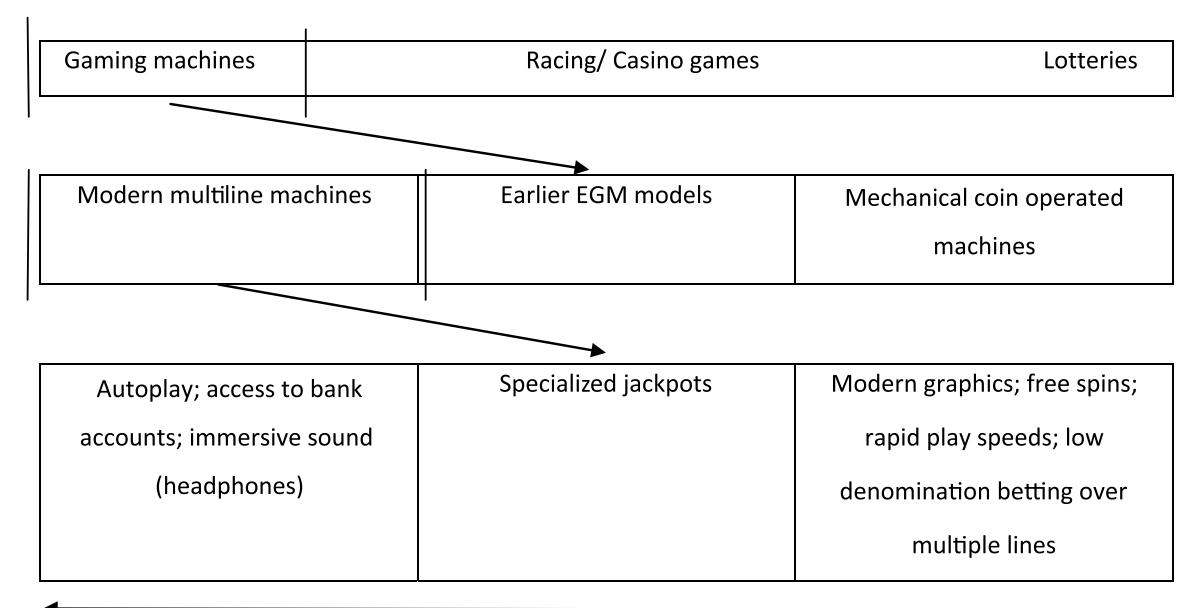

Risk level

Figure 1. Across- versus within-category comparisons of gambling features associated with riskier play and harm

Figure 1 represents a hypothetical, but realistic, depiction of how many researchers would organize the risk associated with different forms of gambling. Activities such as bingo and lotteries would usually be placed on the lower end of the risk continuum (least continuous) and gaming machines at the risky end. Other activities would fall somewhere in between. Policy-makers can avail themselves using tools such as Gamgard (www.gamgard.com) and others to classify the risk associated with various forms of gambling and show that EGMs are a higher risk activity because of multiple features (Line 1). Similarly, it would be possible to predict that modern multiline machines will be more problematic than early generation machines (Line 2). However, the task becomes more difficult as one moves down to Line 3, which represents just the modern market of multiline machines. Nearly all modern EGMs have fast play speeds, intricate graphics and sound, and most may have bonus features and jackpots. At present, although such homogenization still allows meaningful cross category comparisons (e.g., the riskiness of EGMs vs. casino table games), it becomes harder to make useful within the EGM category (e.g., free spin and jackpot features), which is often the main policy focus (e.g., in UK or Australia) (A useful illustrative analogy could be made in gaming. It is known that massive online role-playing games seem to impose greater risks to gamers than other forms of gaming. Thus, if there were a hypothetical world where almost all major video games were of this form, then researchers would be left having to find ways to differentiate between the riskiness of different MMORPGs: a more difficult task). Even tools such as Gamgard may find it hard to identify machines, which are riskier than others. Instead, one would have to look to modify general features common to all the machines rather than look for riskier categories of machine. Such a situation does not undermine and in fact may strengthen experimental research, which looks at the presence or absence of features [e.g., losses disguised as wins (LDWs) or near misses, e.g., Dixon, Harrigan, Sandhu, Collins, \& Fugelsang, 2010], but studies of variations in these features across machines may be harder if all machines have a similar LDW presence. In countries such as the UK and
Australia, where EGM features have been a strong area of public debate, the discussion has typically converged features such as bet sizes or play speeds (i.e., factors that influence the costs of gambling) and whether these can be limited across all machines.

\section{COST AND PLAY DYNAMICS IN EGM GAMBLING}

Much of how we understand EGMs has been influenced by traditional experimental and survey research. Such research generally adopts a static approach that examines the role of individual features in isolation and this serves to establish the importance of individual features in controlled environments. Once again, research tends to adopt either a supplyor demand-side focus. Supply-side approaches draw upon the logic of traditional psychological research in the area of learning theory. The EGM offers a variable or random ratio schedule and the aim is to examine how player behavior varies when aspects of the schedule or reinforcement is modified (Turner \& Horbay, 2004). For example, experiments might be conducted to manipulate the speed of play (Blaszczynski, Sharpe, Walker, Shannon, \& Coughlan, 2005), the return to player (RTP; Haw, 2008), the percentage of LDWs (Dixon et al., 2014), the presence or absence of sound (e.g., Delfabbro, Falzon, \& Ingram, 2005; Dixon et al., 2013; Loba, Stewart, Klein, \& Blackburn, 2001), or the role of losses (Canale, Rubaltelli, Vieno, Pittarello, \& Billieux, 2017). Alternatively, researchers might adopt a demand-side approach and ask gamblers which features they find most appealing or what machines they prefer. For example, there may be questions about their preferred denomination of machine ( 1 cent, 2 cent, and 5 cent per credit); the numbers of lines preferred or how much they bet per line (Delfabbro, 2017).

However, a difficulty with studying EGMs is that the behavioral scenario is not equivalent to the simple laboratory situation involving rats and pigeons. Unlike laboratory schedules that have preprogrammed parameters, EGMs are 
dynamic. The nature of the schedule itself can be modified by the player. Not only does the player have control over how fast he or she responds and the typical reinforcement frequency, players can alter the magnitude of reinforcement. Thus, EGM features, and particularly the important ones relating to the cost of gambling, such as bet sizes, reinforcement frequency, reinforcement size, and play duration, are not independent. When a player changes one feature, he/she will usually change the other. A common scenario that occurs on multiline EGMs is depicted in Figure 2 (The authors acknowledge that there may be a very small percentage of machines in the world, which themselves adapt some of the parameters based on the outcome of the game. We have not included variations in RTP because in many countries like Australia, these fall within a narrow band such that differences are not likely to be discernable by players in typical sessions of play).

In each game, the gambler can make choices about how much he or she wants to bet, how many lines are chosen and therefore the total stake. Increasing the bet size usually increases the magnitude of the reinforcements (bigger win multipliers). Expanding the breath of play by choosing more lines yields more chances to win and therefore more frequent reinforcements. The gambler can, in effect, choose to have a different experience of gambling. Playing more lines or with higher bets will usually lead to shorter sessions of playing for a given budget, but will usually result in either larger or more frequent wins during this period of play.

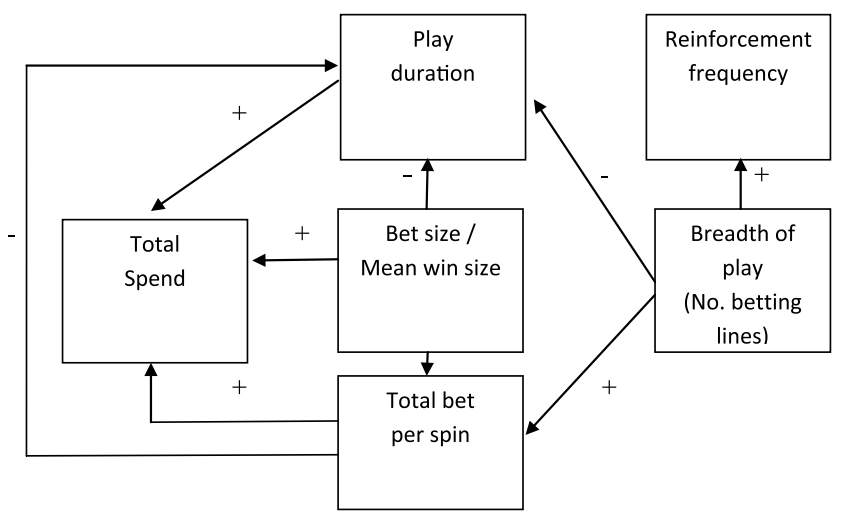

Figure 2. Basic play dynamics of a multiline electronic gaming machine

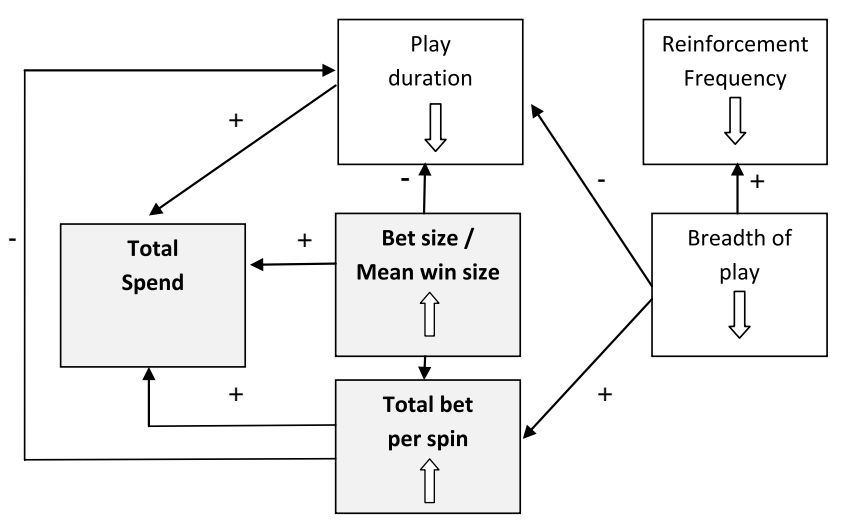

Figure 3. Volatile playing style (assuming fixed budget): larger bets on fewer lines

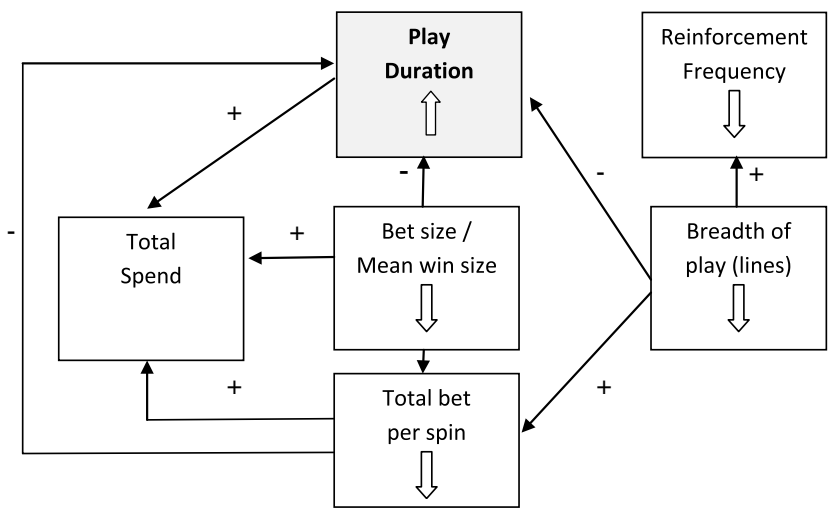

Figure 4. Less volatile playing style (assuming fixed budget): smaller bets on fewer play lines

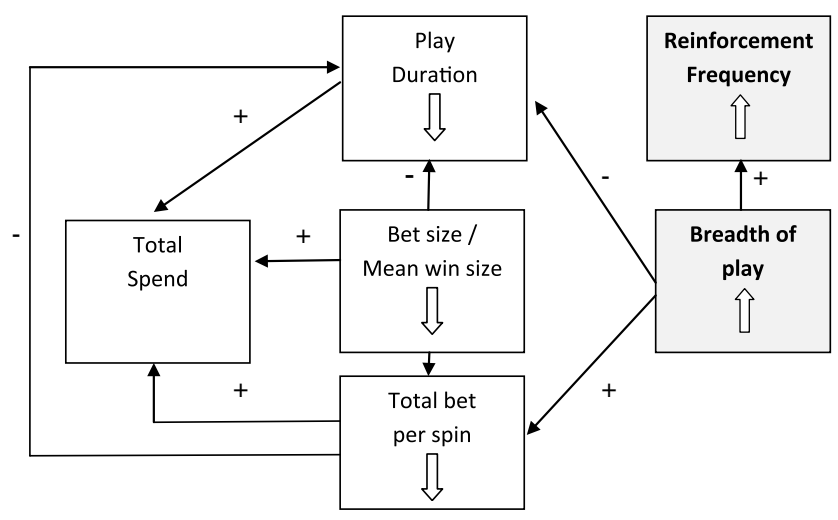

Figure 5. Common low bet/multiline playing style (assuming fixed budget): smaller bets spread over many lines

Some examples of different potential playing styles are depicted in Figures 3-5. Increasing parameters are indicated by bolded text and shading. Figure 3 presents a scenario where a player might choose to increase the mean bet per line, but play on fewer lines. Such a strategy should lead to more volatile outcomes: fewer, but larger wins, longer losing streaks and most likely a reduced play time. Figure 4 represents a situation where the person plays few lines and makes small bets per line. This would typically yield a flat, less volatile style of play characterized by a longer play time, with occasional small wins.

The final example (Figure 5) represents the style of play most commonly adopted by players: what Walker (2004) and Williamson and Walker (2000) have referred to as a maxi-min strategy. Here, the player bets on most, if not all play lines, but at close to the minimum bet. Such a style leads to a steady rate of reinforcement, smaller wins, and a greater likelihood of LDWs (Dixon et al., 2014), although probably shorter play times due to the greater expenditure per bet. Such a strategy yields a less volatile style of play than the reverse strategy in Figure 2 (mini-max). Players prefer this style of play because it decreases their chances of missing out on wins and may increase their chance of obtaining "scatter features" linked to free-spin features (Walker, 2004). Others such as Dow Schüll (2012) and Parke et al. (2015) argue that this style of multiline play yields a smoother playing experience and reduces the 
length of losing streaks. Another view is that multiline play is more existing and yields stronger positive affect (Dixon et al., 2014) and increases the likelihood of gamblers having flowlike experiences or falling into a "zone" in which they block out the world around them (Dow Schüll, 2012).

Irrespective of why gamblers choose particular playing styles, it is clear that EGM gambling is unlike conventional reinforcement schedules. Instead, gamblers choose to play the schedule they prefer and this can be achieved in several ways. The first is by adapting their style of play. Thus, while the same multiline machine might offer opportunities to spend quickly or slowly, or playing for longer or shorter periods, players can choose how they would like to play. They can choose the level of volatility, the rate of reinforcement, and the average size of any wins. A second method is by choosing between games and machines. In the UK, for example, players on the same machine can choose (using a menu system) between slot-machine games and some of these can be more volatile than others (Parke, 2018). On the contrary, in Australia, players have been observed to seek out machines that clearly offer different playing experiences. Such differences are documented, for example, by Livingstone and Woolley (2007) who observed that the most popular South Australian gaming machines fell into two categories. The first type (e.g., Shogun 1 and 2) featured three play lines and $\$ 1$ bets and encouraged higher expenditure and shorter play times; the other, exemplified by Indian Dreaming, featured low denomination multiline playing. On this latter class of machines, gamblers would play a lot longer, but spend much less per game. In other words, higher levels of expenditure would be associated with two quite different playing signatures, but this would not have necessarily been evident if one merely looked at one single parameter of play.

Livingstone and Woolley (2007) argue that these differences between the two types of machine underscore two pathways to higher expenditure. One way is to encourage greater time on device (TOD) and the other is to encourage greater expenditure per unit of time (i.e., Revenue per Active Player in industry parlance or REVPAC). We would argue that such pathways do not necessarily require two different types of machines. Instead, as we have argued, players can adapt their playing style on multiline machines to spend their money in different ways. Some can bet higher amounts and play for shorter periods to obtain larger wins, or they can play low amounts and play longer. Support for this view is evident, for example, in recent analysis conducted on South Australian using data obtained for all machines $(n=14,295)$ across 600 venues (Delfabbro, 2018). A complete monthly data were available concerning the total bets placed, play duration, net revenue earned by the machine, and the machine type. Machines with higher bets per game as well as longer play durations were associated with revenue, but higher bet machines had lower play

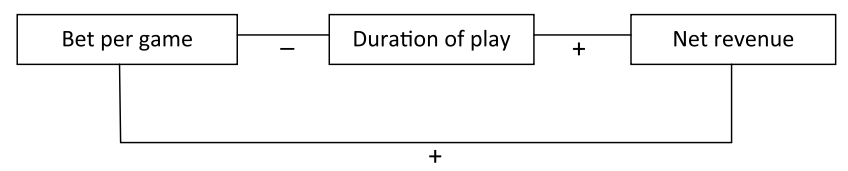

Figure 6. Direct and indirect pathways to greater revenue on EGMs time (as depicted in Figure 6) [This effect was obtained even when analyses were confined to just one type of machine (Dolphin Treasure) with different configurations]. In other words, although it might be tempting to conclude the decreasing or limiting bet sizes will reduce EGM expenditure and revenue (the direct pathway consistent with the REVPAC principle), lower bets also equate to longer sessions, which can also lead to greater expenditure.

In summary, we argue that one may get a better sense of differences between gamblers by placing a greater focus on: (a) analyses that capture the different "play signatures" or styles of players and how these differ according to the status of the gambler and (b) a greater focus on the dynamic interplay between play parameters. For example, it might be possible to detect differences in playing style between higher- and lower-risk gamblers or those with different levels of experience. One likely possibility we anticipate, based on survey studies that have compared the selfreported behavior of infrequent and regular players, is that novice or low-risk gamblers are probably much more likely to choose playing styles consistent with the TOD principle (they play more to have fun and have more game time), whereas higher-risk gamblers, because of their interest in winning, additionally look for ways to increase win size and are attracted by more volatile games or playing experiences (Delfabbro, 2017). This topic is explored in more detail in the following section.

\section{THE DYNAMICS OF PLAYERS: VARIATIONS IN NEED STATES AND MOTIVATION}

It is well documented that people are often attracted to EGMs as a way to fulfill particular needs. Those who develop problems with EGMs share characteristics in consistent with Blaszczynski and Nower's (2002) "vulnerability pathway" into problem gambling or Jacob's (1986) General Theory of Addictions. Such individuals often report clinical levels of depression and anxiety, a history of trauma or abuse, or significant negative life events. Either alone or in combination, these problems create a need to escape or avoid unpleasant mood states, including feelings of sadness, anxiety, and boredom (McCormick, Delfabbro, \& Denson, 2012). By playing EGMs, people are able to locate themselves in an environment where they are separated from the outside world, where they may often be treated with respect by venue staff, and where events may seem, somewhat ironically, more predictable and controllable. In line with this view, Dow Schüll (2012) and Griffiths (1995) have argued that many EGM gamblers enter into a type of psychological "zone" or form of "autopilot" when they gamble. Consistent with some of the symptoms of dissociation, they lose track of time and reality and make even feel that another person is engaging in the activity (Diskin \& Hodgins, 1999, 2001).

Similar views are articulated by Dixon et al. (2014) who refer to a concept called "dark flow," which is described as a mental state in which people are able to disengage from negative thoughts and feelings by immersing themselves into games. In several studies, Dixon et al. (2017) argue that 
dark flow may be a way to compensate for the lack of mindfulness or mental "peace" and self-awareness in everyday life. Using laboratory studies with realistic settings, they found that problem gamblers scored lower on everyday mindfulness, but higher on dark flow. Dark flow was, in turn, found to be positively related to how focused players were on the game within sessions, although this measure was not correlated with problem gambling scores.

A number of authors including Dow Schüll (2012), Dixon et al. (2014), and Parke et al. (2015) suggest that there may be a relationship between the needs of gamblers and the types of gambling experience they seek out. As mentioned above, multiline machines, in particular, are thought to be attractive because they can create a smooth, consistent pattern of reinforcing events (real wins or LDWs) that help maintain the behavior (whether this is described as "the zone" or "dark flow"). Given this observation, it may be important to consider whether this idea of an interaction between the needs of gamblers and their self-selected gambling experiences can be explained more thoroughly. Obviously, EGM gamblers face two competing objectives: one is to stay on the machine and gain satisfaction from the action. The other is to get the outcomes they want (namely, bigger wins or bonus features). However, the more they strive for larger wins, the faster they use up their budget. Conversely, if they merely settle for a low impact, low stakes style of gamble, they get more plays out of the machine, but not necessarily the larger wins they desire. Therefore, it is not hard to see EGM gambling as an activity involving two goals, each of which might be present to varying degrees across players, across sessions, and probably within sessions. A question then arises: Would it be possible for research: (a) to capture which of these objectives are more dominant from people's style of play, (b) to understand whether these motivations influence how people chose to gamble on EGMs, and (c) whether motivations and styles of play might be used to detect higher risk gambling?

This line of thinking is broadly consistent with an important approach to the study of motivations that has been presented in psychology for some years: Apter's (1982) RT. The idea that this theory might be usefully applied to gambling was raised in a paper by Anderson and Brown (1987), but has, since then, largely been ignored. RT is based on the assumption that people's motivations are not static, but dynamic, and like a Necker Cube, switch between states often spontaneously or at the will of the individual. One of the important binary motivation dyads promoted by Apter is the telic: paratelic distinction. A telic mode is one where the person is motivated by the need for the outcome, whereas the paratelic state is principally about the enjoyment or action. As applied to EGM gambling, a telic state would be one where the player was motivated to win. Such gamblers would be expected to look for strategies, winning machines, and find ways to increase winnings. On the contrary, the paratelic gambler would be principally interested in the "action" and the possibility of winning (coming out ahead) would sit in the background (be a secondary aspect to the game and merely what makes it more absorbing).

Anderson and Brown's (1987) analysis suggests that problems arise when there is a conflict between their motivational state and the experiences that they obtain.
If paratelic people are bored and unstimulated, they seek distraction. Those who are telic (and who expect control and goal fulfillment) feel frustrated and anxious when things are not working out the way they expected and they may look for other ways to gain control. Both of these situations can make gambling an attractive activity. In Anderson and Brown's (1987) paper, related to blackjack (a wagering activity), it was assumed that paratelic people would seek excitement and telic people would seek control and a reduction in arousal (the cool-headed, strategic card player). However, given that EGMs are generally accepted to be form of gambling that enables an escape from reality (Dixon, 2018; Dixon et al., 2014; McCormick et al., 2012), it is likely that the paratelic motivation may relate less to elevated arousal, but to a need for soothing absorption and escaping boredom. Moreover, it may be that EGM gambling (because it is very chance-based) always has a strong paratelic element, but that there are people who are more often in a telic state than others (more telic dominant). In other words, EGMs may provide an attractive activity for people for slightly different, but related reasons: reducing boredom and/or also reexerting a sense of predictability and control. Both start in a state of restless agitation (which has a negative hedonic tone) and they reduce this by gambling. In our view, a recent work by Dixon et al.'s (2014) on the concept of "dark flow" is sufficiently inclusive to capture both of these elements.

However, RT suggests that, in real-life gambling (where real losses can be obtained), dark flow could be broken. For example, if players who commence gambling with a more telic motivation start losing, this increases anxiety and encourages a greater focus on winning and chasing behavior that could be best achieved by adopting a playing style where bets are increased at the expense of longer periods of anticipated play time. These views are consistent with the works of Corless and Dickerson (1989) and Gehring and Willoughby (2002) who argue that people may display greater impaired control and risk-taking when agitated and faced with large number of losses. On the other hand, those who gamble to escape may feel bored if the anticipated wins are not obtained or if their play time is cut short by a string of losses. According to RT, fluctuations of mood and behavior may occur multiple times during long gambling sessions and that both winning and losing events could potentially serve to maintain behavior. The problem gambler in a paratelic state cannot stop when he or she craves the psychologically soothing winning periods, but neither can they stop when they start losing. The telic problem gambler wants to feel a sense of predictability and control by obtaining a desired win, but may not stop until this is achieved. This view is supported by research that shows that problem gamblers score much higher on measures of chasing (O'Connor \& Dickerson, 2003) and are more likely to endorse erroneous beliefs about their chances of winning on EGMs such as the gambler's fallacy (Ladouceur, 2004; Lambos \& Delfabbro, 2007; Toneatto, Blitz-Miller, Calderwood, Dragonetti, \& Tsannos, 1997; Toplak, Liu, Macpherson, Toneatto, \& Stanovich, 2007).

If there is merit in this theoretical argument, then it may be possible in research to use techniques (e.g., phone-based apps or body worn sensors) to capture these differences. 
For example, it may be possible to capture whether people are commencing gambling sessions in a state of anxiety (e.g., where arousal is elevated and gamblers are self-rating themselves as not being in a positive mood). Such measurements could be captured during gambling sessions. Such work would potentially allow research to examine whether states of agitation (or problems with mindfulness) lead not only to a desire for Dixon et al.'s (2014) “dark flow," but also measurable differences in the behavior observed, in particular, how people respond to losing sequences. In Dixon's (2018) recent work, the researchers captured within-session ratings of whether people were "thinking about the game" or staying "on task." Higher scores on this measure were related to dark flow but not problem gambling. Is it possible that this measure could be further refined to capture differences in the way in which they were "thinking about the game?" Consistent with RT, it may be that there is more than one motivational pathway underlying a desire for dark flow-type experiences: one that is principally about absorption and escape and another that relates more strongly to reexerting control. It may be that more telic-oriented gamblers are most at risk of chasing because they do not like to lose; their attempts at achieving control in every life have been thwarted and so they look for ways to win on slot machines. Accordingly, it could be hypothesized that a measure that captured thoughts of this nature within sessions might be related to problem gambling as well as dark flow, to the extent that dark flow captures people's desire to reassert control and certainly. A second hypothesis would then be whether one might observe different playing styles for those who have stronger telic orientations. One prediction is that these people will be less concerned with time on the machine, but more interested in winning, so that machines that offer volatility and the chance of a big win may be more attractive than those that offer a slower, more drawn out experience.

\section{CONCLUSIONS AND IMPLICATIONS}

The principal purpose of this paper was to draw attention to the dynamic and complex nature of EGM gambling. Although we see merit in much of the research that have attempted to isolate the influence of individual game parameters, our view is that there are limits to this approach. Modern gaming activities experience a form of "technical momentum" in which yesterday's new features quickly become standard features, so that specific features that increase the risk become something of a moving target. Thus, it becomes increasingly difficult to isolate features, which stand out as ones to address as viable methods of harm reduction or minimization. This problem is further compounded by the complex interplay between different features, so that changes to one feature (e.g., slowing reel speeds or reducing bet sizes) may not necessarily reduce expenditure if they can be offset by another unmodified parameter.

We believe that these observations have implications for both research as well as policy interventions relating to harm-minimization strategies to reduce the harm associated with EGMs. Indeed, as is gradually happening in the United Kingdom (Parke et al., 2015), we believe that greater policy and funding should be directed toward research that tries to capture the interplay between gaming machine features and studies of features in isolation. In particular, we argue that policy may need to take more of a "whole of machine" approach, which looks at the style of play that machines offer and to look at changes that might have a more meaningful impact on high-risk play. In our view, greater attention should be directed toward patterns of behavior or what we have termed "play signatures" rather than just responses to single features, although we recognize that high-quality laboratory studies are essential to providing insights into which individual features might be important in their own right. Such work, we envision, would focus more strongly on actual gambling data that are both longitudinal and also capable of capturing different play elements simultaneously (e.g., whether the gambler is losing during the session, the frequency of reinforcement, betting options chosen, and the volatility of the game). Such analyses may be made possible using modern technological advances in the analysis of big data, but would require greater policy focus on the availability of industryheld data for research purposes.

At a research level, we also argue that there may be value in forging stronger links between research into people's vulnerabilities and motivations and the nature of their behavior. Some important developments of this nature are emerging (e.g., Dixon, 2018; Dixon et al., 2014) and we believe that this is an area with considerable potential. For example, we argue that it may be possible to identify presession psychological states (e.g., presession arousal and anxiety) that might place people at greater risk of excessive gambling using what are "ecological momentary assessments" (e.g., via smartphones) and then relate this to subsequent behavior. One of the important perspectives we believe to emerge from psychological theory (RT) is that maladaptive or harmful gambling may be momentarily predictable. A gambler who attempts to gamble while anxious or distressed and where there is a goal-orientation (telic state) directed toward winning on EGMs is more likely to be at risk of harm. Such players, we predict, will (a) adopt a playing style or choose machines where behavior will be more goal-oriented toward winning as opposed to merely enjoyment and (b) respond differently to periods of losing. Potential associations of this nature between need states and behavior therefore raise the possibility that there may be useful devices or apps that might be developed to assist higher-risk gamblers in situ (e.g., the recently developed Curb Your Urge App in Australia; Merkouris, Dowling, Hawker, Rodda, \& Youssef, 2018). These could be used as "secondary" interventions to help gamblers who may be at risk of harm or be applied in clinical treatment programs that involve controlled gambling and exposure to gambling locations.

Funding sources: This paper did not receive any funding.

Authors' contribution: Both the authors made significant contributions to the drafting of the paper. 
Conflict of interest: Dr. DLK had previously undertaken research funded by Victorian Responsible Gambling Foundation, Gambling Research Australia, and received travel support from the WHO. Prof. PD had undertaken research funded by Gambling Research Australia, Victorian Responsible Gambling Foundation, and the other parts of the Victorian State Government. He had also undertaken reviews for GambleAware (UK), Canadian research funding bodies (Manitoba, AGRI), and travel support to present at the International Regulators Conferences (IAGR). The authors report no conflict of interest in preparation of this manuscript.

\section{REFERENCES}

Anderson, G., \& Brown, R. I. F. (1987). Some applications of Reversal Theory to the explanation of gambling and gambling addictions. Journal of Gambling Behavior, 3(3), 179-189. doi:10.1007/BF01367439

Apter, M. J. (1982). The experience of motivation: The theory of psychological reversals. London, UK: Academic Press.

Armstrong, A., \& Carroll, M. (2017). Gambling activity in Australia. Melbourne, Australia: Australian Gambling Research Centre, Australian Institute of Family Studies.

Armstrong, A. R., Thomas, A., \& Abbott, M. (2017). Gambling participation, expenditure and risk of harm in Australia, 1997-98 and 2010-11. Journal of Gambling Studies, 34(1), 255-274. doi:10.1007/s10899-017-9708-0

Blaszczynski, A., \& Nower, L. (2002). A pathways model of problem and pathological gambling. Addiction, 97(5), 487-499. doi:10.1046/j.1360-0443.2002.00015.x

Blaszczynski, A., Sharpe, L., Walker, M., Shannon, K., \& Coughlan, M.-J. (2005). Structural characteristics of electronic gaming machines and satisfaction of play among recreational and problem gamblers. International Gambling Studies, 5(2), 187-198. doi:10.1080/14459790500303378

Canale, N., Rubaltelli, E., Vieno, A., Pittarello, A., \& Billieux, J. (2017). Impulsivity influences betting under stress in laboratory gambling. Science Reports, 7(1), 10668. doi:10.1038/s41598-017-10745-9

Corless, A., \& Dickerson, M. G. (1989). Gambler's self-perceptions of determinants of impaired control. British Journal of Addiction, 84(12), 1527-1537. doi:10.1111/j.1360-0443.1989.tb03936.x

Delfabbro, P. H. (2017). Australasian gambling review. Adelaide, Australia: Independent Gambling Authority.

Delfabbro, P. H. (2018). Addiction by default?: Analysis of EGM characteristics and their association with gambling behavior. Paper presented at the 12th European Conference on Gambling Studies and Policy Issues, Valletta, Malta.

Delfabbro, P. H., Falzon, K., \& Ingram, T. (2005). The effects of parameter variations in electronic gambling simulations: Results of a laboratory-based pilot study. Gambling Research, 17(1), 7-25.

Diskin, K. M., \& Hodgins, D. C. (1999). Narrowing of attention and dissociation in pathological video lottery gamblers. Journal of Gambling Studies, 15(1), 17-28.

Diskin, K. M., \& Hodgins, D.C.(2001). Narrowed focus and dissociative experiences in a community sample of experienced video lottery gamblers. Canadian Journal of Behavioural Science, 33(1), 58-64. doi: $10.1037 / \mathrm{h} 0087128$
Dixon, M. J. (2018). Dark flow and reactivity to rewards: Distinct routes to slot machine enjoyment for problem and recreational slots players. Paper presented at the 17th Annual Alberta Gambling Research Institute, Banff, Canada.

Dixon, M. J., Harrigan, K. A., Sandhu, R., Collins, K., \& Fugelsang, J. A. (2010). Losses disguised as wins in modern multi-line video slot machines. Addiction, 105(10), 1819-1824. doi:10.1111/j.1360-0443.2010.03050.x

Dixon, M. J., Harrigan, K., Santessa, D. L., Graydon, C., Fugelsang, J., \& Collins, K. (2013). The impact of sound in modern multiline video slot machines. Journal of Gambling Studies, 30(4), 913-929. doi:10.1007/s10899-013-9391-8

Dixon, M. J., Harrigan, K. A., Santesso, D. L., Graydon, C., Fugelsang, J. A., \& Collins, K. (2014). The impact of sound in modern multiline video slot machine play. Journal of Gambling Studies, 30(4), 913-929. doi:10.1007/s10899-013-9391-8

Dixon, M. J., Stange, M., Larche, C., Graydon, C., Fuselsang, J., \& Harrigan, K. (2017). Dark flow, depression and multiline slot machine play. Journal of Gambling Studies, 34, 73-84. doi:10.1007/s10899-017-9695-1

Doran, B., \& Young, M. (2010). Predicting the spatial distribution of gambling vulnerability: An application of gravity modeling using ABS Mesh Blocks. Applied Geography, 30(1), 141-152. doi:10.1016/j.apgeog.2009.04.002

Dowling, N., Smith, D., \& Thomas, T. (2005). Electronic gaming machines: Are they the 'crack-cocaine' of gambling? Journal of Addiction, 100(1), 33-45. doi:10.1111/j.1360-0443.2005.00962.x

Dow Schüll, N. (2012). Addiction by design. Princeton, NJ: Princeton University Press.

Gehring, W. J., \& Willoughby, A. R. (2002). The medial frontal cortex and the rapid processing of monetary gains and losses. Science, 295(5563), 2279-2282. doi:10.1126/science.1066893

Griffiths, M. D. (1995). Adolescent gambling. London, UK/ New York, NY: Routledge.

Haw, J. (2008). The relationship between reinforcement and gaming machine choice. Journal of Gambling Studies, 24(1), 55-61. doi:10.1007/s10899-007-9073-5

Jacobs, D. (1986). A general theory of addictions: A new theoretical model. Journal of Gambling Behavior, 2(1), 15-31. doi:10.1007/BF01019931

Ladouceur, R. (2004). Perceptions among pathological and nonpathological gamblers. Addictive Behaviours, 29(3), 555-565. doi:10.1016/j.addbeh.2003.08.025

Lambos, C., \& Delfabbro, P. H. (2007). Numerical reasoning ability and irrational beliefs in problem gambling. International Gambling Studies, 7(2), 157-171. doi:10.1080/14459790 701387428

Livingstone, C. (2001). The social economy of poker machine gambling in Victoria. International Gambling Studies, 1(1), 46-65. doi:10.1080/14459800108732287

Livingstone, C. (2005). Desire and the consumption of danger: Electronic gaming machines and the commodification of inferiority. Addiction Research and Theory, 13(6), 523-534. doi:10.1080/16066350500338161

Livingstone, C., \& Woolley, R. (2007). Risky business: A few provocations on the regulation of electronic gaming machines. International Gambling Studies, 7(3), 361-376. doi:10.1080/ 14459790701601810

Livingstone, C., Woolley, R., Zazryn, T., Bakacs, L., \& Shami, R. (2008). The relevance and role of gaming machine games and 
game features on the play of problem gamblers. Adelaide, Australia: Independent Gambling Authority.

Loba, P., Stewart, S. H., Klein, R. M., \& Blackburn, J. R. (2001). Manipulations of the features of standard video lottery terminal (VLT) games: Effects in pathological and non-pathological gamblers. Journal of Gambling Studies, 17(4), 297-320. doi:10.1023/A:1013639729908

McCormick, J., Delfabbro, P., \& Denson, L. (2012). Psychological vulnerability and problem gambling: An application of Durand Jacobs' general theory of addictions to electronic gaming machine playing in Australia. Journal of Gambling Studies, 28(4), 665-690. doi:10.1007/s10899-011-9281-x

Merkouris, S., Dowling, N., Hawker, C., Rodda, S., \& Youssef, G. (2018). Gamblingless Curb Your Urge: Development and user testing of a smartphone intervention application for problem gambling. Paper presented at the National Association for Gambling Studies Conference, Brisbane, Australia.

Neal, P., Delfabbro, P. H., \& O’Neil, M. (2005). Problem gambling and harm: Towards a national definition. Adelaide, Australia: Centre for Economic Studies.

O'Connor, J. \& Dickerson, M. (2003). Definition and measure of chasing in off-course betting and gaming machine play. Journal of Gambling Studies, 19(4), 359-386.

Parke, J. (2018). Got game? Taking stock of 50 years of research in product-related risk and looking ahead beyond 2020. Paper presented at the 12th European Conference on Gambling Studies and Policy Issues, Valletta, Malta.

Parke, J., Parke, A., \& Blaszczynski, A. (2015). Key issues in product-based harm minimisation. London, UK: The Responsible Gambling Trust.
Productivity Commission. (1999). Australia's gambling industries. Canberra, Australia: Productivity Commission.

Productivity Commission. (2010). Gambling. Canberra, Australia: Productivity Commission.

Queensland Treasury. (2018). Australian gambling statistics (34th ed.). Brisbane, Australia: Queensland Government Statistician's Office.

Toneatto, T., Blitz-Miller, T., Calderwood, K., Dragonetti, R., \& Tsannos, A. (1997). Cognitive distortions in heavy gambling. Journal of Gambling Studies, 13(3), 253-266. doi:10.1023/ A:1024983300428

Toplak, M. E., Liu, E., Macpherson, R., Toneatto, T., \& Stanovich, K. (2007). The reasoning skills and thinking dispositions of problem gamblers: A dual-process taxonomy. Journal of Behavioural Decision Making, 20(2), 103-124. doi:10.1002/ bdm.544

Turner, N., \& Horbay, R. (2004). How do slot machines and electronic gaming machines actually work? Journal of Gambling Issues, 11, 1-42.

Walker, M. B. (2004). The seductiveness of poker machines. Gambling Research, 16, 52-66.

Williamson, A., \& Walker, M. (2000). Strategies for solving the insoluble: Playing to win Queen of the Nile. In G. Coman (Ed.), Lessons of the past: Proceedings of the 10th Annual Conference of the National Association for Gambling Studies (pp. 444-452). Mildura, Victoria: National Association for Gambling Studies.

Ziolkowski, S. (2016). The world count of gaming machines 2015. Sydney, Australia: Gaming Technology Association. Retrieved from www.gamingta.com 